Cahiers de recherches médiévales

\title{
La manipulation littéraire de la femme féerique dans Les Prophesies de Merlin
}

Portrait d'une « mégère inapprivoisée »

\section{Virginie Derrien}

\section{OpenEdition \\ Journals}

Édition électronique

URL : https://journals.openedition.org/crm/5543

DOI : $10.4000 / \mathrm{crm} .5543$

ISSN : 1955-2424

Éditeur

Honoré Champion

Édition imprimée

Date de publication : 20 juin 2008

Pagination : 19-30

ISSN : 1272-9752

Référence électronique

Virginie Derrien, "La manipulation littéraire de la femme féerique dans Les Prophesies de Merlin »,

Cahiers de recherches médiévales [En ligne], 15 | 2008, mis en ligne le 20 juin 2011, consulté le 15

décembre 2022. URL : http://journals.openedition.org/crm/5543; DOl : https://doi.org/10.4000/crm. 5543 


\title{
㮣M
}

\section{La manipulation littéraire de la femme féerique dans les Prophe- sies de Merlin : portrait d'une « mégère inapprivoisée »}

\begin{abstract}
Les Prophesies de Merlin is a propitious text for an inquiry of the marvellous in the late Middle Ages, and highlights, beyond an aesthetic effect, the essence of romance writing that had developed in the course of this transitory period, thus paving the way for works of Rabelais and Cerventes. This literary work from the $14^{\text {th }}$ century provides a degenerated depiction of the female characters who, in earlier Arthurian literature, are represented as the most beautiful and powerful fairies, and perverts the patterns from earlier romance texts, so as to produce a comical perspective of an upside-down world, which borders on the fabliau genre.
\end{abstract}

Résumé : Les Prophesies de Merlin sont un texte propice à l'étude du merveilleux des romans du Moyen Âge tardif, en ce qu'il révèle, au-delà d'un effet esthétique, l'essence d'une écriture romanesque qui a pris son essor au cours de cette époque charnière, anticipant ainsi sur les oeuvres de Rabelais et de Cerventes. Ce texte littéraire du XIV siècle propose un portrait décadent des personnages féminins - qui, dans la littérature arthurienne antérieure, incarnaient les fées les plus belles et les plus puissantes - de manière à inscrire l'écriture des motifs traditionnels dans une perspective comique qui conduit le lecteur aux confins du genre des fabliaux.

Dans l'immense tapisserie que déploient les Prophesies de Merlin', constituée de prédictions essentiellement politiques ${ }^{2}$, certains épisodes narratifs empruntés au corpus arthurien fonctionnent comme des micro-récits et peuvent faire l'objet d'une lecture autonome. Lucy Allen Paton situe la date de composition du manuscrit Mss. Maggs Brothers, support de l'édition d'Anne Berthelot sur laquelle se fonde notre étude et qui appartient dorénavant à la Fondation Bodmer, au début du XIV siècle. L'auteur du manuscrit de Bodmer, au sein de ces insertions romanesques, réserve une place de choix aux dames faées. Les romans du Moyen Âge tardif reposent sur un manichéisme bien défini qu'exemplifient les personnages relevant du merveilleux : le système des personnages féminins en particulier est fondé sur une division interne puisque les dames féeriques rivalisent avec les magiciennes, ces dernières mettant leurs pouvoirs au service du mal. C'est dans le cadre de cette répartition que se définit la femme merveilleuse dans Les Prophesies de Merlin, texte qui propose une réécriture intéressante de ce motif littéraire.

${ }^{1}$ Les Prophesies de Merlin, éd. A. Berthelot, Cologny-Genève, Fondation Martin Bodmer, 1992. Voir également l'introduction de L. Allen Paton, Les Prophecies de Merlin, D.C. Heath and Compagny, New York, Oxford University Press, London, 1926, Vol. I, p. 9.

${ }^{2}$ Voir l'introduction d'A. Berthelot à l'édition, p. 9-10.

Cahiers de Recherches Médiévales, 15, 2008 
Les fées, survivantes d'ancestrales croyances, sont à l'origine des femmes humaines qui, en bénéficiant d'un apprentissage particulier, peuvent pratiquer les arts magiques: dames instruites, elles détiennent leur savoir de Merlin dont l'origine diabolique peut justifier que certaines d'entre elles se tournent vers le mal. La bonne Dame du Lac s'efforcera de remplacer Merlin (symbole de l'Antéchrist qu'il fallait éliminer) auprès d'Arthur, et s'oppose donc à Morgue, dont la présence implique irrémédiablement, ainsi que le mentionne Anne Berthelot, «magie, danger et aventures sulfureuses $»^{3}$. La mutation de Morgue, initialement fée venue de l'Autre Monde pour ravir le cœur d'un mortel, en femme savante mettant sa science au service d'une luxure condamnable marque la prose arthurienne du XIII ${ }^{\mathrm{e}}$ siècle. Morgue vise la destruction du monde d'Arthur et de ses valeurs, tandis que La Dame du Lac incarne l'éloge de l'amour courtois et de la chevalerie $e^{4}$. Elles sont ainsi deux figures antithétiques dans un jeu de forces qui constitue la structure de la prose arthurienne qui les met en scène.

Loin d'être un motif éculé, l'opposition entre ces dames surgit dans les Prophesies de Merlin: leurs caractéristiques divergentes développées par la tradition régissent leur traitement et leur rôle au sein de ce texte tardif dans lequel le «trinôme» Merlin-Morgue-Dame du Lac fonctionne toujours. Au cours de l'un de ses entretiens avec Antoine, chargé de mettre par écrit ses prophéties, Merlin avoue son penchant pour La Dame du Lac. C'est au cours de ces effusions que Merlin dresse un portrait traditionnel de la fée en effectuant une comparaison entre sa « favorite» et la rivale de celle-ci :

- Di moi Mierlin se Diex te saut, fait maistre Antoine, la quele des dames ke tu as conneue par mi le monde as tu trouvee la plus sage des autres ? - Or met en ton escrit, cou dist Mierlins, ke se toutes les femmes del monde estoient a une part, ke pour naturel sens, ke pour l'art de clergie, pour toute le soutillite del monde, et la dame del Lac fust d'autre part me tenroie iou avant a li que a toutes les autres. [...] Dieus ayde, cou dist maistres Antoine, dont est ele plus sage ke n'est Morghe ? Taisies, sire, cou dist Mierlins, que iou croi vraiment ke cele Morghe nasqui dou fu de luxure. Et de la biele dame dou Lac croi iou que ele nasqui auques pries de paradis. Cele Morghe porcace et fait les males oeures. Et cele del lac fait les boines oeures. Cele fait ocire les bons chevaliers et cele les fais secorre et aidier. Cele fera

\footnotetext{
3 «La Dame du Lac, Sébile l'enchanteresse, la dame d'Avalon ... et quelques autres », Mélanges Wolfgang Spiewok, Greifswald, Reineke-Verlag, 1994, p. 15. A. Berthelot étudie la survie littéraire des fées (les traditionnelles figures comme Morgue et La Dame du Lac, ou les «petites nouvelles » comme Sébile et la Dame d'Avalon...) dans les Prophesies de Merlin, les relations qu'elles entretiennent, leurs rapports avec leur maître Merlin. Elle parvient à la conclusion que ces magiciennes sont le moyen d'apporter «un peu de sang neuf dont [le roman arthurien] a tant besoin, dans la mesure où [ces nouveaux] personnages servent de supports à de nouvelles aventures -ou à des aventures anciennes déguisées au goût du jour », p. 16.

${ }^{4}$ Voir J-M Boivin, «La Dame du Lac, Morgane et Galehaut. Symbolique de trois figures emblématiques de l'Autre Monde dans le Lancelot ", Médiévales, nº. Printemps 1984, p. 18 25 .
} 
encore secourre les orphelins et cele Morghe les fera destruire en despit de lor peres et de lors meres. Prophesies de Merlin (p. 70-71)

Tout est dit, ou plutôt répété, et la tradition demeure opérationnelle dans l'esprit du lecteur: il n'y a pas nécessité de s'expliquer sur les origines de ces personnages, et la narration se contente d'insister plutôt sur leur fonction respective au sein de la société arthurienne. Mais le dénominateur commun et le plus révélateur demeure Merlin lui-même, en ce qu'il est à l'origine de leur «formation scientifique »: nul besoin de rappeler que Merlin manifeste un penchant très prononcé pour les séduisantes dames qui se présentent à lui. C'est sans conteste cette fâcheuse tendance qui le voue à sa perte dans tous les textes qui le mentionnent. Bien qu'il ne puisse déterminer l'heure de sa mort ni qui lui fera expier ses péchés de chair, Merlin n'est pas dupe car ses qualités de prophète lui permettent de prédire sa fin (p. 70). Mais Merlin, qui avait prophétisé qu'une blance serpente [le] decheveroit (p. 94), ne peut imaginer que c'est sa bonne amie, dont il pense avoir gagné l'estime, qui l'attend pour lui tendre un piège. Car si Mierlins amoit la Dame del Lac a tout son pooir et de tout son cuer, la dame le haoit autant ou plus (p.92). La Dame du Lac lui témoigne toutes les marques d'affection qu'elle peut feindre, et l'invite à entrer dans la tombe qu'elle lui a préparée afin de constater si l'espace est suffisamment grand pour deux corps ${ }^{5}$ :

Et quant la Dame del Lac ki a cou l'avoit mene le vit gissant dedens la tombe ele en abati erranment le couviercle, et fist maintenant l'esperiement, et tantost fu la tombe tant bien fremee et dedens et dehors, einsi com il meismes li avoit apris, que nus hom del monde, tant fust sages, ki dedens ne dehors le peust desfremer, ne tant ne quant. (p. 94-95)

On note, à l'instar des textes antérieurs, l'ironie de la situation : la fin dérisoire du plus célèbre magicien est persistante car, une fois encore, le prophèteenchanteur ne peut échapper à son sort : non seulement il est incapable de deviner que la fée le condamne à une réclusion définitive, mais le maître se voit fait prisonnier par les enchantements que lui-même a transmis à son élève préférée. Merlin reproche alors à La Dame du Lac son action en ce qu'elle fausse la prophétie le concernant et selon laquelle il serait perdu par une «blance serpente». Mais La Dame du Lac le rassure immédiatement sur ses dons de prédictions en lui expliquant qu'en aucun cas Merlin ne lui a pris sa virginité : elle s'était protégée grâce aux premiers enseignements que Merlin lui avait dispensés et qui consistaient à endormir ou réveiller un homme selon son bon vouloir (p. 95). Cependant, les raisons pour lesquelles Merlin est entombé concernent non seulement le désir de La Dame du Lac de préserver sa chasteté mais aussi de sauver sa réputation que son ennemie de toujours se plaît à ternir, ainsi qu'elle l'explique à son prisonnier :

${ }^{5}$ Merlin exprime en effet son désir que La Dame du Lac, après sa mort, soit déposée à ses côtés, p. 94. 
Saces tout vraiement ke jou t'ai mis ichi dedens pour cou que tu aloies disant en tous les lius ou tu aloies ke tu avoies geu a moi, et si en fui pute clamee par la bouce meismes Morghain. (p. 95)

Dans ce texte tardif, c'est toujours l'amour propre qui est à l'origine de l'entombement de Merlin, mais le motif prend une tonalité parodique: la chaste marraine de Lancelot commet ce "crime d'honneur» pour empêcher Merlin de colporter des mensonges sur leur relation, mettre ainsi un terme aux moqueries de Morgue, et surtout séduire le chevalier Méliadus dont elle est tombée amoureuse. On remarque ainsi un effet de lecture déviant par rapport au matériau littéraire antérieur puisque le rôle de marraine féerique qui a fait la renommée de La Dame du Lac dans la prose du XIII ${ }^{\mathrm{e}}$ siècle est à peine développé dans le texte; il est moins question d'une figure maternelle au merveilleux bénéfique, qui fera don à la cour d'Arthur d'un héros doté de pouvoirs surhumains, que d'une femme humaine, humiliée, qui agit pour assouvir sa vengeance, faire taire les «mauvaises langues » et gagner les faveurs de son ami Méliadus en lui prouvant qu'elle n'a rien perdu de son intégrité.

L'auteur adopte l'attitude amorcée au siècle précédent et qui consiste à intégrer l'être merveilleux dans le récit au moyen d'une rationalisation : tout ce qui provient de l'Autre Monde, qui dérive d'un surnaturel païen, doit être adapté aux contraintes qu'impose le système chrétien qui régit les mentalités de l'époque ${ }^{6}$. Mais outre cette technique de banalisation, qui permet de confondre l'image de la fée avec celle d'une dame courtoise et réaliste, notre auteur procède à d'autres ajustements de mises en récit du merveilleux ; la figure merveilleuse vise à servir de nouveaux desseins sémantiques et littéraires. La déviation vers le burlesque (au sens où la représentation même de ces fées, leur langage, leur comportement prennent un aspect ridicule qui contraste avec leur portrait canonique prévalant dans les romans antérieurs) devient l'un des procédés d'écriture largement exploité dans les Prophesies de Merlin. La dérision, le traitement humoristique contaminent de manière pratiquement générale les épisodes dans lesquels les femmes faées sont au premier plan. L'un des épisodes les plus emblématiques est celui qui confronte Morgue la fée et Sébile l'enchanteresse, dont le nom renvoie le lecteur à l'antique Sybille de l'Enéas . Mais là encore, on comprendra que les termes de fée et d'enchanteresse sont utilisés par l'énonciation pour mieux être mis à distance tant le sémantisme qui leur est ordinairement attribué s'en trouve perverti. Nos dames féeriques ne s'engagent plus au sein d'une lutte entre forces merveilleuses bénéfiques et puissances maléfiques, pour ou contre les principes de la fin'amor ou de la Chevalerie, mais se livrent à des querelles de chiffonnières. La violence qui les anime éclaire non plus les tensions qui les opposaient au sein d'une organisation politique constituée par la cour

\footnotetext{
${ }^{6}$ Voir A. Berthelot, «Magiciennes et enchanteurs : Comment apprivoiser l'autre faé », Chants et enchantements au Moyen Âge, Lectures Médiévales, Université de Toulouse II, Champion, 1997, p. 105-120.

${ }^{7}$ Enéas, éd. J. J. Salverda de Grave, Paris, Champion, 1973, voir v. 2199-2209. Sybille, chaste prophétesse et «Sage Prêtresse», représentait le savoir du monde antique au service d'œuvres bénéfiques.
} 
d'Arthur, mais dénoncent plutôt les rapports (de force) entre les femmes. Elles ne se font concurrence que pour obtenir le cœur d'un homme, et la rivalité amoureuse entre ces dames est racontée avec verve. Sébile s'éprend de Bielenger le Beau qui recherche désespérément son fils. L'enchanteresse promet à Bielenger de le lui ramener s'il accepte de l'épouser. Le marché se conclut, et le couple se rend chez Morgue, qui détient l'enfant. C'était bien entendu sans compter sur la luxure de cette dernière qui, de toute évidence, tombe sous le charme du beau Bielenger. Morgue feint d'aider Sébile mais demande à l'une de ses demoiselles de faire savoir à Bielenger qu'il ne récupèrera pas son fils. La promesse de mariage avec Sébile sera rompue, et Fleur de Lys pourra en obtenir une autre, selon la coutume du guerredon, mais cette fois-ci pour le compte de sa maîtresse. Cependant Morgue se fait doubler par Fleur de Lys, qui recueille finalement les faveurs de Bielenger, et s'enfuit avec lui et l'enfant. Dès le lendemain matin, lorsque les deux magiciennes découvrent la disparition du chevalier sur lequel elles avaient l'une et l'autre jeté leur dévolu, une vive querelle éclate entre elles. Sébile fait part à Morgue de ses soupçons de trahison, puisqu'elle pense à juste titre que sa comparse est à l'origine de quelques manigances pour obtenir la préférence de Bielenger :

- Car iou vous sent a si desloial et a si luxurieuse et a si putain ke iou le me pensai par maintes fois en ceste nuit; car onques mais ne vous poi tant proier ke de vostre bouce en issist nule boine parole. - Ha! Pute vachiere, fait Morghe, adont m'aves putain clamee! (p. 210).

Sébile, par ses insultes, n'apprend rien de bien nouveau au lecteur, habitué aux frasques de Morgue. Mais la réécriture du motif est frappante, innovante, en ce qu'elle s'inspire du style des fabliaux. Si le genre burlesque ne s'établit en France qu'au XVII ${ }^{\mathrm{e}}$ siècle, on remarque cependant que notre texte s'y prête allègrement. Les prémisses de ce genre littéraire apparaissent dans la manière de travestir le roman arthurien considéré comme noble, en prêtant aux héroïnes faées des actions et des propos vulgaires et bas : non seulement nos femmes faées, ou du moins ce qu'il en reste, s'expriment en un langage grossier peu digne des fées à l'aura poétique des œuvres précédentes, mais elles se battent également comme des furies :

Et lors li court sus Sébile mout vistement. Quant ele vit que ele li couru sus, ele prist erraument Morghain par les chaviax et l'abat a terre. [...] Morghe estoit auques d'eage, si ne pot avoir viers Sébile duree, dont ele le traina par mi les treces, or cha, or la, par mi la sale et li doune souventes fois dou piet en mi la gorge et de sor le nes $[\ldots]$ et manace toutes voies mout durement ke se ele ne li rent son mari que a la mort le metra. (p. 210)

Ainsi que le suggère Philippe Ménard, «pour se divertir du spectacle, il suffit d'estimer que tous les combattants appartiennent à un monde bas et vulgaire et de juger qu'ils méritent d'être maltraités $\gg{ }^{8}$. C'est donc la lecture que nous proposons de cette scène, puisque l'humour se situe dans le regard adopté par le narrateur qui

${ }^{8}$ Les Fabliaux : contes à rire du Moyen Âge, Paris, PUF, 1983, p. 203. 
choisit d'appréhender la figure féerique au travers d'un prisme réducteur: la force physique déployée par Sébile affecte l'identité autrefois «super-féerique» de Morgue, en réduisant cette dernière à un simple corps que l'on peut aisément rouer de coups. Notre auteur procure à la fois un portrait de Morgue hérité de ses prédécesseurs, et une déformation de l'image du personnage de la femme merveilleuse en procédant à une réduction des codes intratextuels, en perturbant les références du lecteur. Pour rendre l'humiliation de Morgue comique, la narration a pris le soin de déprécier la victime de la brutalité de Sébile: les déboires de Morgue, «putain» de l'histoire et par conséquent disqualifiée moralement, peuvent donc prêter au sourire. L'image de la plus cruelle enchanteresse se voit gauchie, sa grandeur étant entachée par cette situation humiliante: Morgue, sous la douleur, crie et brait, et n'en réchappe que grâce à l'intervention de ses serviteurs. Au moyen de cet affrontement, l'auteur nous livre ainsi une nouvelle version de la femme merveilleuse: Morgue, puissante enchanteresse démoniaque, coupable des pires trahisons qui ont provoqué la chute du monde arthurien dans la prose du XIII ${ }^{\mathrm{e}}$ siècle ${ }^{9}$, est considérée dans ce texte comme une bien piètre magicienne. Il n'est plus question de recourir à l'art des enchantements pour retenir l'amant dans l'Autre Monde et réduire celui-ci à sa merci car, au contraire, Morgue s'en remet aux ruses de l'esprit en demandant à sa suivante de mentir à Bielenger. D'ailleurs, la sulfureuse fée semble avoir perdu de son prestige puisque le chevalier décide de s'enfuir avec sa demoiselle. Oubliée aussi la «nigremance» pour terrasser ses adversaires puisqu'elle a besoin de l'intervention de ses serviteurs pour échapper aux coups de Sébile. Morgue se voit aussi réduite à solliciter l'aide de la reine de Norgales pour obtenir vengeance. Cette requête, par le biais d'une lettre, permet une fois encore de ramener la figure de la fée à celle d'une dame aux contours plus que réalistes, voire prosaïques : Morgue propose de coucher la reine de Norgales sur son testament en échange de son secours. Ce nouveau chantage est le moyen d'introduire le comique de ton et de situation dans le récit : très certainement pour la première fois depuis son apparition dans la littérature, Morgue, défigurée, avoue avoir été humiliée et manifeste une générosité sans égal :

- Sire capelains, or me faites erranment unes letres ; salues moi la roine de Norgales de par Morghain, laz trainee par toute la sale par les treches, et batue et defoulee et mise en sanc et le vis enflet, la bouce et le nes et les iex; veoir le viegne anscois ke ele muire. Et se ele le veut vengier de sa honte, sace certainement ke iou li laisserai mes livres et mes mars, et ke apries ma mort les pora prendre comme siens ; et encore li ferai iou plus, ke iou ne me metrai en saisine dou chastiel de Norgales. (p.226)

${ }^{9}$ Voir E. Vinaver, «La fée Morgue et les aventures de Bretagne », Mélanges J. Frappier, t. 2, 1970, p. 1081-82: «Sans renoncer à son rôle de motif additionnel, [la fée Morgue] évoque, sur le plan romanesque, l'ornatus difficilis des poétiques médiévales. Conçue en triptyque, elle donne lieu à trois séries d'épisodes respectivement centrés sur Urien, Arthur et Accalon. [...] Tout se ramène en fin de compte au panneau central, au sort d'Arthur menacé par la magicienne qui a déjà fait périr son amant, qui a levé le bras sur son mari et qui prépare de loin la mort de son frère. [...] Ceux que la fée Morgain honore de sa haine ne vivent pas longtemps ». 
Dans cet extrait, le processus de rationalisation de la figure faée atteint son apogée, car Morgue perd ici toute trace de beauté, de féerie, d'immortalité et de poésie pour incarner une mégère enlaidie, la régente d'un fief qui planifiait l'extension de son domaine. La magie qui auparavant servait les grands desseins eschatologiques dans la prose arthurienne se voit, dans ce roman tardif, supplantée par des intérêts financiers individuels; la poésie qui émanait des paroles des dames merveilleuses n'a plus lieu d'être car dorénavant on leur attribue un langage ordurier ou juridique et économique. Si la production romanesque du XIII ${ }^{\mathrm{e}}$ siècle avait opté pour les procédés de démonisation ou de christianisation afin de justifier la présence de l'être faé dans le récit, la littérature tardive choisit la méthode d'évhémérisation, voire de prosaïsation pour dissimuler tous les signes surnaturels: le camouflage fonctionne, et ce traitement drastique nous propose le portrait d'une fée essentiellement caractérisée par la mesquinerie et la concupiscence. Cependant Morgue, malgré cette proposition alléchante, n'obtient pas l'assentiment de la reine de Norgales : cette dernière se rend au chevet de $\mathrm{Morgue}^{10}$, et fait prendre conscience à son interlocutrice qu'en aucun cas elle ne peut faire porter le blâme à Sébile. Elle rappelle un épisode dans lequel Morgue et sa complice Sébile l'avaient éloignée de son amant afin que les deux comparses puissent passer la nuit avec lui. Morgue abdique alors devant la force de conviction des propos de la reine, et reconnaît elle même ses torts (p. 228). Dès lors Sébile peut se présenter devant Morgue, et les trois enchanteresses sont à nouveau réconciliées. On saisit les enjeux littéraires du surnaturel aux yeux de l'auteur puisque la femme merveilleuse devient un instrument au service du comique, et le burlesque vient colorer les épisodes qui la font intervenir. Nos fées au comportement grotesque permettent de détourner le genre arthurien vers la parodie, maintes fois exploitée par certains épisodes qui ne manquent pas de panache et méritent d'être racontés pour leur piquant. L'auteur prend le parti de rendre ces femmes ridicules en organisant un véritable «meeting » d'enchanteresses. Morgue, Sébile et la reine de Norgales se rendent chez la Dame d'Avalon ${ }^{11}$, qui a convoqué ses collègues pour éprouver leurs pouvoirs face aux anneaux magiques que lui a ramenés Eglantine. Dès que la Dame d'Avalon passe l'un des anneaux à son doigt, bien fu avis a tous chiaus ki el palais estoient que li monde fust venus al definer (p. 340). Sébile et la reine de Norgales ont beau jeter elles aussi leurs enchantements pour faire face à cette illusion d'apocalypse mais rien n'y fait. Au contraire la Dame d'Avalon, témoin d'une panique générale, décide de se moquer de ses hôtes:

\footnotetext{
${ }^{10}$ Scène qui prête également au sourire tant Morgue joue les martyrs pour susciter la compassion de la reine : «Quant la nouviele fu venue a Morghain ke la roine le venoit veoir, ele fist samblant ke ele eust asses pis ke ele n'avoit», p. 227.

${ }^{11}$ Nous précisons que Sébile et la reine de Norgales, invitées les premières, se dirigent vers le château où réside Morgue afin que celle-ci se joigne à elles. Mais lorsque que Morgue « oi la nouviele que ans .ii. les dames planes d'encantement estoient descendues, ele ot paour de traison par coi ele se fit celer», p. 339. Nous remarquons une fois encore que dès que l'occasion se présente, l'auteur n'hésite jamais à dévaloriser Morgue, à lui ôter toute l'assurance et la superbe qui la définissaient dans les œuvres antérieures.
} 
Et lors se mist Sébile avant, si gieta son encantement, dont il fu avis a tous chiaus ki laiens estoient que li fus fust entres el palais. Et lors prissent errant lor dras et lor lis et lor autre harnois, si le getierent aval. Et quant la dame d'Avalon vit cou, ele traist erranment cel anelet hors de son doit et i mist .i. autre, et puis vint a la roine de Norgales, si li dist : «Dame, ostes vos dras hors de votre dos », et puis dist ele autretel a Sébile. La force de la piere de l'analet les amena maintenant a cou que eles osterent erranment tous lor dras hors de lors dos, et puis les getierent aval ensi com la dame d'Avalon le requist. [...] Et quant les ars furent faillies et les .ii. dames se virent toutes nues, se eles orent honte cou ne fait pas a demander. (p. 340)

Le comique non seulement consiste en une dépréciation de la figure de la fée et de sa personnalité, mais se glisse également dans l'illusion générée à la fois par la tromperie intradiégétique que créent ces enchantements, et par la chimère que produit ce texte littéraire lui-même. L'enchantement provoque une illusion captieuse qui amenuise les capacités de jugement des victimes, et engendre un comique de situation: les enchanteresses tombent dans le piège qui leur est tendu et, croyant se débarrasser des draps, se défont de leurs vêtements. La facétie, liée au vocabulaire de la déception (il fu avis), modifie autant le système de référence sur lequel repose le comportement de nos enchanteresses malmenées, que celui du lecteur confronté à une nouvelle logique textuelle. Si les auteurs de la prose du XIII ${ }^{\mathrm{e}}$ siècle s'évertuent à identifier les êtres surnaturels issus de croyances païennes à des femmes humaines pour justifier leur présence et leur rôle au sein de la société arthurienne, celui des Prophesies de Merlin prend le parti de les déshumaniser et de les désocialiser : nos dites fées perdent leur humanité en se séparant de leur apparat vestimentaire ${ }^{12}$. Le vêtement est le signe d'un état, d'une condition sociale, et participe en cela de l'identité féminine ou masculine ${ }^{13}$. Telle une armure qui détermine le statut chevaleresque d'un homme et protège son corps des coups de ses adversaires au cours des combats, la robe souligne les courbes du corps féminin qu'elle met en valeur tout en dissimulant les formes interdites au regard. Dépossédées de leur robe, nos dames apparaissent alors désarmées, incapables de masquer leur nudité et de préserver leur intégrité physique et morale. La Dame d'Avalon, qui ainsi réussit à soumettre ses adversaires et à les rallier à ses côtés, espère pouvoir en faire de même avec Morgue (p. 340). L'attente ne sera pas longue, car Morgue, qui se rit de l'humiliation endurée par ses compères, ne tarde pas à arriver en " grande pompe", en convoquant une legion des anemis d'infier, probablement pour impressionner la Dame d'Avalon. Mais les «diablotins » envoyés par Morgue s'avèrent inutiles et impuissants pour capturer la Dame d'Avalon, protégée par ses anneaux. Et c'est ainsi que la Dame

${ }^{12}$ Voir E. J. Burns, «Refashioning Courtly Love», in Constructing Medieval Sexuality, Eds. K. Lochrie, P. Mc Cracken, J. A. Schultz, Medieval Cultures, vol. 11, University of Minnesota Press, Minneapolis, 1997, p. 111-134: «Rather than having the status of some matter or ground on which cultural constructions are built, the courtly body can be understood in this light as a set of clothes that make, mark, delimit, and define the body presumed to lie beneath », p. 113-114.

${ }^{13}$ Voir J. Le Goff, « Codes vestimentaire et alimentaire dans Erec et Enide », Pour un autre Moyen Âge, Paris, Gallimard, 1999, p. 615-633. 
d'Avalon et par la même occasion le lecteur peuvent savourer la défaite de Morgue, racontée pour son comique propre : Morgue prise au piège de l'illusion fallacieuse et surtout facétieuse, à l'instar de ses prédécesseurs, obéit à l'injonction de son hôtesse en lui donnant ce qu'elle croit être des draps et se retrouve bien évidemment toute nue, situation humiliante qui accule Morgue à s'avouer vaincue :

- Ha ! Dame, fait Morge, honnie m'aves, car on quidoit que iou fuisce de petit eage, et il ont veue ma char, et mes mamieles pendillans, et la piel de mon ventre contreval la tierre, dont la parole serra en maint liu contee d'or en avant. (p. 342)

Plus encore que les coups infligés précédemment par Sébile, c'est l'inversion coercitive et les conséquences aliénantes sur Morgue, dépourvue de capacité de raisonnement, qui font sourire : en faisant (une fois encore) de Morgue une victime, l'auteur dévalue l'image traditionnelle de la fée, réduite à un corps vieillissant et dénué de toute séduction. La nudité de Morgue, référence symbolique, est l'expression d'une vérité appréhendée selon l'équivalence entre beauté corporelle et perfection morale. Morgue, ainsi révélée à l'état brut, ne s'offre plus au regard comme l'expression d'une merveille (la belle fée désirée) mais expose un physique rebutant, signe de sa perfidie, conformément à l'esthétique médiévale qui associe le Beau au Bien et le Laid au Mal ${ }^{14}$.

Cette scène prête également au sourire grâce aux conséquences burlesques qui dépouillent le motif de l'anneau magique de son sens symbolique et de la dimension courtoise à laquelle il est généralement associé. L'anneau était auparavant un auxiliaire magique au service de la quête chevaleresque et un symbole de la féminité. On pense, par exemple, à l'anneau que La Dame du Lac offre à Lancelot en lui expliquant ses vertus, à savoir que cet objet a tel forche qu'il descuevre tous enchantemens et fait veoir ${ }^{15}$. L'anneau, donné par amour ${ }^{16}$, remplit une fonction protectrice en ce qu'il permet au chevalier de ne pas céder aux enchantements et de mener à bien ses aventures. Mais rien de tel dans les Prophésies de Merlin, texte qui se plaît à prendre à rebours les motifs traditionnels de la fin'amor et de l'exaltation chevaleresque. Le motif de l'anneau dans cet épisode devient le prétexte d'une scène de « nigremance» à grand renfort d'effets spéciaux et dont la seule fonction consiste à tourner en dérision la rivalité entre ces magiciennes, en quelque sorte, peu compétentes. Morgue et ses consœurs se lamentent sur leur sort et regrettent Merlin, seul enchanteur habilité en la matière. Convaincues qu'elles pourraient apprendre davantage de lui afin de ne plus revivre ce genre d'humiliation, elles se lancent à sa re-

\footnotetext{
${ }^{14}$ Voir J. Le Goff, «Corps et Idéologie dans l'Occident Médiéval», Pour un autre Moyen Âge, op. cit., p. 555-558: «Quant aux clivages sociaux laïcs essentiels ils ne s'expriment jamais mieux qu'en oppositions corporelles : le noble est beau et bien fait, le vilain est laid et difforme », p. 556.

${ }^{15}$ Lancelot, roman en prose du XIII siècle, éd. A Micha, Paris-Genève, Droz, 1978-1983, t. VII, p. 270. Sur la fonction de l'anneau dans le roman arthurien, voir Jean-René Valette, $L a$ Poétique du merveilleux dans le Lancelot en prose, Paris, Champion, 1998, p. 331-333.

${ }^{16} \mathrm{C}$ 'est le cas de Guenièvre qui remet à Lancelot un anneau «le jor qu'ele li dona s'amor », et qui permet à son amant de remporter l'épreuve du Val sans Retour, t. I, p. 290.
} 
cherche $^{17}$. L'auteur se joue donc de ses femmes merveilleuses pour le plaisir des lecteurs et le sien: la crainte générée par les diables convoqués par Morgue se trouve vite désamorcée par un comique de situation et de mots, qui confère une tonalité parodique à l'affrontement entre les magiciennes, traité de façon grotesque. Les femmes faées, metteurs en scène de situations comiques, insufflent au récit arthurien un élan de bouffonnerie. Grâce à nos dames, avatars burlesques des traditionnelles fées, l'auteur souligne que les valeurs courtoises, qui régissaient la société arthurienne et constituaient l'essence chevaleresque, sont devenues obsolètes : cette dégradation morale qui transparaît dans la réécriture du motif de la fée suggère une évolution des structures mentales en cette fin de Moyen Âge, et répond probablement à l'attente du lecteur dont la perception des figures littéraires, et le cas échéant merveilleuses, marque un tournant dans la production romanesque : au XIII ${ }^{\mathrm{e}}$ siècle, le merveilleux est mystique et pessimiste; les créatures maléfiques y sont démonisées pour être ensuite éliminées ; les êtres surnaturels bénéfiques, et par conséquent les fées, doivent être rationalisés pour obtenir un droit de cité dans l'oeuvre; seules les merveilles procédant du merveilleux chrétien sont tolérées ${ }^{18}$ et le symbolisme religieux prédomine. Mais la chute du royaume arthurien dans la Mort Artu entraîne avec elle les «merveilles de Bretagne», et la production romanesque qui suit doit trouver le moyen de renouveler le surnaturel, son écriture et son interprétation. Dès lors, il ne s'agit plus seulement de normaliser l'être surnaturel mais de le ridiculiser, moyen d'instruire en divertissant. Sous ce vernis de décadence et de grotesque résonne une voix satirique qui oriente l'interprétation morale que l'on peut tirer de ces épisodes : c'est la situation de la femme faée (mais qu'il est douloureux de l'appeler toujours ainsi !) aux prises avec ses désirs, sa sexualité débridée, ses mesquineries, en un mot ses péchés, qui prime. L'auteur procède à une dévalorisation contextuelle de celles qu'il prend pour victimes: victimes intertextuelles en ce que les dames féeriques se font fi de leur rôle traditionnel et utilisent le peu de pouvoirs qu'il leur est imparti pour se mesurer et s'humilier entre elles; victimes extratextuelles, puisque dotées de tares physiques (on pense à la version enlaidie de Morgue) et de travers psychologiques, l'auteur leur refuse toute once de sensibilité et d'intelligence pour mieux accentuer un tableau de femmes qui portent en elles l'idée de déchéance. Les Prophésies de Merlin, texte fidèle à son ambition didactique, incitent ainsi le

\footnotetext{
${ }^{17}$ Voir A. Berthelot, «La Dame du Lac, Sébile l'enchanteresse, la dame d'Avalon ... et quelques autres », art. cit., p. $13:$ «[...] elles doivent admettre [que Merlin] leur manque non pas sur le plan affectif, mais d'un point de vue purement intéressé : il était vraiment le meilleur! Elles décident d'aller à sa recherche, initiant ainsi une quête pour Merlin qui malheureusement tourne court, mais semble originellement conçue comme un pendant, ou une parodie, de la Quête du Graal». On le comprend, chaque initiative de la part des magiciennes est vouée à l'échec, et permet, en jouant sur l'intertextualité, de réécrire le matériau connu selon une perspective parodique. Le lecteur s'y retrouve aisément en identifiant motifs et caractères, et sa lecture se trouve satisfaite et comblée de par la réécriture déformée, la variation qui apporte une nouvelle tonalité (la touche humoristique) au récit arthurien.

${ }^{18}$ Voir F. Dubost, «Entre (ir)rationalité et symbolisme : la merveille », Théophilyon, II 2, 1997, p. 475.
} 
lecteur à réfléchir sur sa condition, sa relation aux femmes. À plusieurs reprises le récit insiste sur les causes de la disparition de Merlin, à savoir son penchant exacerbé pour les jolies demoiselles dénuées de tous scrupules. C'est la voix de Merlin elle-même, depuis son tombeau, qui profère une sévère opinion à l'encontre des femmes (p. 109-110). L'emblème d'une telle critique se cristallise dans le personnage de Morgue, tributaire de sa fonction traditionnelle. Sa réputation de «séductrice fatale » à la fin du Moyen Âge n'est plus à faire, et l'auteur récupère cette caractéristique pour mieux accentuer son dessein littéraire et proposer une critique acerbe de la manipulation féminine : coutumière de retenir prisonniers les hommes qu'elle veut pour amants, Morgue détient au moyen d'une drogue le bel Alexandre. Une demoiselle explique ainsi au chevalier la raison de sa captivité :

- Et si voel que vous sachies que [Morgue] ne vous retient pour autre cosse fors seulement pour sooler sa luxure. [...] sachies que tous ses travaux ne tous ses espoirs n'est se pour sa luxure non saouler. (p. 359)

Mais Alexandre, grâce à l'aide de la demoiselle, finit par s'échapper, et Morgue, une fois de plus, verra ses désirs frustrés. La construction littéraire de la figure féerique repose sur une poétique de l'excès et du manque : les besoins excessifs et coupables de la fée ne peuvent être comblés. L'auteur se complaît ainsi dans l'abjection de la représentation de la femme, qu'il prive de pouvoirs dans leur plus large acception : la femme est démunie de pouvoirs magiques, séducteurs/sexuels ${ }^{19}$, et politiques puisqu'elle ne se voit conférer aucun rôle dans l'organisation arthurienne. Un récit tel que les Prophésies de Merlin, sous le couvert des grivoiseries de fabliaux, permet de débattre sur ce que la morale, la société et la religion interdisent. Le texte, en modifiant l'essence même de la dame féerique, peut fonctionner comme une satire de la femme: l'auteur ne met pas en scène des figures merveilleuses qui incarnent un idéal courtois au service d'une classe chevaleresque, mais donne vie à des dames qui infirment les remarquables qualités habituellement attribuées au fées. En dépouillant le topos de l'enchanteresse des artifices séducteurs que propose la féerie traditionnelle, l'auteur crée des dames plus conformes à la réalité (ou du moins à sa réalité) en ce qu'elles ne sont plus infaillibles et s'avouent victimes de leurs faiblesses.

L'intérêt des scènes aux colorations arthuriennes de ce texte tardif réside en un gauchissement très patent à travers la mise en scène de la femme merveilleuse. Cette technique littéraire correspond peut-être à l'horizon d'attente du lectorat médiéval ${ }^{20}$, dont la réception d'un texte reposerait sur cet " écart esthétique ${ }^{21}$ qui vient

\footnotetext{
${ }^{19}$ Lorsque la demoiselle de Morgue explique à Alexandre pourquoi sa maîtresse le retient prisonnier, celui-ci s'empresse de répondre: «Et tant voel iou que vous sachies, fait li Orfenins Alixandres, que ancois que iou couchaisse od si vielle dame et o si laide com est Morghe trenceroie iou a mes .ii. mains ans .ii. pendans », p. 359.

${ }^{20}$ Voir H-R Jauss, Pour une esthétique de la réception, Paris, Gallimard, 1978, p. 53 : «La façon dont une oeuvre littéraire, au moment où elle apparaît, répond à l'attente de son premier public, la dépasse, la déçoit ou la contredit, fournit évidemment un critère pour le jugement de
} 
troubler les expectations établies par de précédentes lectures. Les Prophésies de Merlin nous propulsent dans un monde aux conventions bouleversées, signe que les enjeux de l'écriture romanesque ont évolué : enchantements et merveilleux sont le moyen d'introduire et de légitimer dans ce roman des scènes de théâtre de rue. La malléabilité qu'autorise l'écriture du surnaturel permet d'enrichir la narration, et de renouveler l'esthétique littéraire: nos dames faées, précurseurs des personnages de Rabelais, s'expriment comme des mégères, se chamaillent comme des chiffonnières et se contentent de brasser du vent, probablement pour permettre aux moulins à vent de Cerventès de tourner.

Virginie Derrien

Aberystwyth

sa valeur esthétique. L'écart entre l'horizon d'attente et l'oeuvre, entre ce que l'expérience esthétique antérieure offre de familier et le «changement d'horizon » [requis] par l'accueil de la nouvelle oeuvre, détermine, pour l'esthétique de la réception, le caractère proprement artistique d'une oeuvre littéraire ».

${ }^{21}$ Ibid., p. 53 : «On appelle écart esthétique la distance entre l'horizon d'attente préexistant et l'oeuvre nouvelle dont la réception peut entraîner un "changement d'horizon » en allant à l'encontre d'expériences familières ». 